\title{
Preliminary results from XMM-Newton observations of the massive Wolf-Rayet binary WR 22 (WN7h+O9III-V)
}

\author{
Eric Gosset, Gregor Rauw, Jean-Marie Vreux, Yaël Nazé, \\ Igor I. Antokhin, and Hugues Sana \\ Institut d'Astrophysique et de Géophysique, Université de Liège, \\ 17 Allée du 6 août, B-4000 Liège 1 (Sart-Tilman), la Belgique
}

\begin{abstract}
We present preliminary results from observations of the massive WR+O binary WR 22 performed with the XMM-Newton observatory. The star is brighter in the X-ray domain near apastron and fainter at periastron. The change in brightness is very progressive and mainly corresponds to a lowering of the soft part of the spectrum.
\end{abstract}

\section{Introduction}

WR 22 (HD 92740) is a bright WR+O binary system ( $P=80.3$ days) located in Carina OB1. The system, in an eccentric orbit, hosts the most massive WolfRayet star ever weighed. The WN7h component has been assigned a minimum mass of $72 \mathrm{M}_{\odot}$ (Rauw et al. 1996) or of $55 \mathrm{M}_{\odot}$ (Schweickhardt et al. 1999). The mass ratio $M_{\mathrm{WN}} / M_{\mathrm{O}}=2.7$. Such a system is expected to exhibit a colliding wind phenomenon dominated by the WR wind.

Both components, as well as the probable shock zone, are susceptible to emit X-rays. The first detection of WR 22 in the X-ray domain was reported by Seward \& Chlebowski (1982) as part of an investigation of the Carina region with the Einstein satellite. Pollock (1987) reported of three observations of WR 22 with Einstein (two near apastron and one near periastron) and concluded that they were clearly suggesting that the X-ray luminosity varies with the orbital cycle, the system being brighter near apastron (near the lower conjunction of the O-type star).

In the present paper, we report preliminary results from recent observations of WR 22 with the ESA satellite XMM-Newton (Jansen et al. 2001).

\section{Observations}

WR 22 was monitored in the X-ray domain with the ESA XMM-Newton observatory in the framework of the GT programme. Seven $10 \mathrm{ksec}$ (nominal) pointings regularly distributed in orbital phase between apastron and periastron were scheduled. Only the two XMM-EPIC-MOS camera results are treated here. Some of the pointings suffered from soft proton flares; the exposure corresponding to the apastron is particularly affected by the phenomenon.

For WR 22, the source count rates as well as the background were estimated over specific energy bands for each individual pointing. 


\section{Results}

The resulting mean count rates per sec per MOS (in the band $\sim 0.5-5.0 \mathrm{keV}$ ) are $0.033(\phi=0.50$ near apastron), $0.040(\phi=0.58), 0.038(\phi=0.68), 0.031$ $(\phi=0.77), 0.031(\phi=0.83), 0.024(\phi=0.90)$ and $0.016(\phi=0.995$, near periastron and within the optical eclipse, see Gosset et al. 1991). The error is of the order of 0.005 or less, except for the apastron pointing where it is three times larger. The count rates clearly indicate that WR 22 is variable along its orbital cycle. With the exception of the apastron, the variation appears rather smooth with a progressive decrease from phase $\phi=0.58$ to phase $\phi=0.995$. In the energy range $0.5-5.0 \mathrm{keV}$, the count rates at periastron only represent $40 \%$ of those at phase $\phi=0.58$. This ratio compares well with the Einstein count rates behaviour reported by Pollock (1987) for the $0.2-4 \mathrm{keV}$ band.

This behaviour is quite different from the case of HD 93403 (O5.5I+O7V, see Rauw et al. 2002) and is much more reminiscent of $\gamma^{2}$ Vel (WR 11, WC8+O7.5III$\mathrm{V}$, see Dumm et al. 2002), where the low opacity inside the cone-shaped cavity of the interacting winds is supposed to explain the peaked hard X-ray emission when the O-type star is in front of the WR star in the line-of-sight.

In order to further investigate the phenomenon, we partitioned the observed count rates in three energy bands $(0.5-1.0 \mathrm{keV}, 1.0-2.0 \mathrm{keV}$, and $2.0-5.0 \mathrm{keV})$. The decrease in the X-ray flux with the increasing phase is essentially due to the loss of soft X-ray photons, the flux in the hardest band being almost constant. The O-type star could contribute to the soft X-ray component. Our observations are thus highly suggestive of a progressive extinction of the X-ray emission through the WR wind opacity. If true, this means that WR 22 offers the rare opportunity to radially scan the wind opacity of a WR star at X-ray energies by observing it along its orbital cycle.

Besides WR 22 itself, the combined X-ray image of the field reveals a few tens of point sources as well as a clear diffuse emission occupying a large fraction of the field.

\section{References}

Dumm, Th., Güdel, M., Schmutz, W., Audard, M., Schild, H., Leutenegger, M., van der Hucht, K.A. 2002, in: F. Jansen et al. (eds.), New Visions of the X-ray Universe in the XMM-Newton and Chandra Era, ESA SP-488, in press

Gosset, E., Remy, M., Manfroid, J., Vreux, J.-M., Balona, L.A., Sterken, C., Franco, G.A.P. 1991, IBVS 3571, 1

Jansen, F., Lumb, D., Altieri, B., et al. 2001, A\&A (Letters) 365, L1

Pollock, A.M.T. 1987, ApJ 320, 283

Rauw, G., Vreux, J.-M., Gosset, E., Hutsemékers, D., Magain, P., Rochowicz, K. 1996, A\&A 306, 771

Rauw, G., Vreux, J.-M., Stevens, I.R., Gosset, E., Sana, H., Jamar, C., Mason, K.O. 2002, A\&A 388, 552

Schweickhardt, J., Schmutz, W., Stahl, O., Szeifert, Th., Wolf, B. 1999, A\&A 347, 127

Seward, F.D., Chlebowski, T. 1982, ApJ 256, 530 